Dorota Masiakowska-Osses

ORCID: 0000-0001-9592-4174

Adam-Mickiewicz-Universität, Poznań

DOI: 10.19195/0435-5865.143.6

\title{
„Wer an die Zukunft denkt, muss sich erinnern können." Deniz Utlus Roman Die Ungehaltenen zum Gedenken an die Vätergeneration
}

\begin{abstract}
s
Der vorliegende Beitrag untersucht Deniz Utlus Debütroman aus dem Jahre 2014, u.d. T. Die Ungehaltenen. Seine Protagonisten sind zwei junge Berliner aus dem Einwanderungsviertel Kreuzberg, Kinder der türkischen „Gastarbeiter“ der ersten Stunde. Ihre Väter sterben alt und siech, unterschätzt und marginalisiert von der deutschen Gesellschaft. Ausgehend von dem Konzept des kulturellen Gedächtnisses wird gezeigt, auf welche Art und Weise Utlu angesichts des Ablebens von Zeugen die Erinnerung an die erste Generation der türkischen Nachkriegsmigranten fördert und mitgestaltet, indem er unter anderem auf die Lokalgeschichte Kreuzbergs, Berlins Einwanderungsstadtteils per se, zurückgreift. Darüber hinaus wird auch auf Utlus Kritik der Modi des Erinnerns und der medialen Repräsentation der Migrationsgeschichte in Deutschland eingegangen (Jubiläen, Musealisierung).
\end{abstract}

Schlüsselwörter: türkisch-deutsche Literatur, Deniz Utlu, „Gastarbeiter“, kulturelles Gedächtnis, Migrationsgeschichte, mediale Repräsentation der Geschichte

\section{"Who thinks about the future must be able to remember". Deniz Utlu's novel Die Ungehaltenen in the memory of the fathers' generation}

This paper analyses the debut novel by Deniz Utlu, published in 2014 under the title Die Ungehaltenen [The Indignant]. The novel's protagonists are two Berliners from Kreuzberg, children of Turkish "guest workers", who are facing sickness and death while feeling marginalised and underestimated. Using the concept of cultural memory, the author shows how Utlu's novel saves from oblivion and co-shapes the memory of the first postwar generation of Turkish immigrants in the face of the death of witnesses, in particular by appealing to the history of Kreuzberg - an immigrant district of Berlin per se. The analysis also touches upon the critical reflection detected in the novel on the ways of

\footnotetext{
${ }^{1}$ Utlu (2011).
} 
commemorating and representing the history of immigrants in Germany by the media (celebrating anniversaries, musealisation).

Keywords: Turkish-German literature, Deniz Utlu, "guest workers", cultural memory, history of immigration, representation of history in the media

Dorota Masiakowska-Osses, Uniwersytet im. Adama Mickiewicza, Instytut Filologii Germańskiej, Al. Niepodległości 4, 61-874 Poznań, Polen, E-Mail: domaska@amu.edu.pl

Received: 30.09.2017, accepted: 4.05.2018

\section{Das kulturelle Gedächtnis und die Einwanderung}

In dem kanonisch gewordenen Werk Das kulturelle Gedächtnis. Schrift, Erinnerung und politische Identität in frühen Hochkulturen unterscheidet Jan Assmann zwischen dem kommunikativen und dem kulturellen Gedächtnis. Das kommunikative Gedächtnis erfasst nach ihm biografische Erinnerungen aus der jüngsten Vergangenheit, die mit Zeitgenossen geteilt und in meist informellen Interaktionen, oft im Familienkreis, vermittelt werden (J. Assmann 2007: 50). Diese naturwüchsige Art von Gedächtnis vergeht auch natürlicherweise mit seinen Trägern. Nachdem die letzten Zeugen gestorben sind und der unmittelbare Erfahrungshorizont verschwunden ist, tritt an die Stelle des kommunikativen das kulturelle Gedächtnis. Es „richtet sich auf Fixpunkte in der Vergangenheit“, wobei sich diese zu „symbolischen Figuren“"verdichten, ,an die sich die Erinnerung heftet“ (J. Assmann 2007: 52).

Obwohl Jan Assmann (2007: 51) den Zeithorizont der lebendigen Erinnerung auf achtzig bis hundert Jahre, also drei, vier Generationen schätzt, scheinen ihm vierzig Jahre eine „kritische Schwelle“ zu bilden. Da nach ungefähr dieser Zeit die Zeitzeugen aus dem Berufsleben ausscheiden, wachsen in ihnen die Erinnerungen und der Wunsch nach deren Fixierung und Weitergabe. Soll diesem Wunsch nachgekommen werden, muss hier die Erinnerungsarbeit, vor allem die Speicherung, einsetzen, sowohl seitens der Zeitzeugen einer Erinnerungsgemeinschaft als auch seitens der spezialisierten Traditionsträger der Gesellschaft, zum Beispiel der Archivare.

Mit der Speicherung ist zwar eine Voraussetzung, aber keine Garantie gegeben, dass das Erinnerte zum Bestandteil des kulturellen Gedächtnisses einer Nation wird und nicht dem sozialen Vergessen zum Opfer fällt. In der Beschreibung des kulturellen Gedächtnisses spricht Jan Assmann (2007: 54 f.) von Einweisungen, Kontrolle der Verbreitung und strikten Grenzen, die darum gezogen werden. Denn Erinnern ist ihm zufolge ein Prozess sowohl der Sinnstiftung als auch der Selektion: „Nur bedeutsame Vergangenheit wird erinnert, nur erinnerte Vergangenheit wird bedeutsam“ (2007: 77). Als bedeutsam gilt wiederum das, was 
die Identität der erinnernden Gruppe fundiert und zu erhalten hilft. Auch Aleida Assmann hält die Identitätsvergewisserung - neben ethischer Verpflichtung und Neugier - für eines der zentralen Motive sowohl der individuellen als auch der nationalen Hinwendung zur Geschichte (A. Assmann 2007: 25 ff.).

Im Falle einer nationalen Gemeinschaft werden im Fundus der kollektiven Erinnerungen solche als potenziell identitätsstiftend angesehen, die von der Mehrheit ihrer Mitglieder geteilt und der Herstellung einer Kontinuität dienen können. Dass sich die Erinnerungen der Einwanderer nicht nahtlos in dieses Mehrheitsnarrativ einfügen, ist offensichtlich. Ob beziehungsweise unter welchen Bedingungen sie sich nicht doch einfügen ließen, ist eine andere Frage, die unter anderem die postmigrantische Literatur zunehmend beschäftigt. Denn im Prinzip ist das kulturelle Gedächtnis auch als Konstrukt etwas Offenes, etwas im Wandel Begriffenes. Aleida Assmann schätzt den Rhythmus eines grundlegenden Wandels des Gedächtnisses einer Gesellschaft auf dreißig Jahre. Im abschließenden Teil ihres Werkes Geschichte im Gedächtnis stellt sie eine zuwanderungsbedingte Heterogenisierung der Bevölkerung fest und mahnt: „Bei der Wiederfindung der Nation im Medium der Geschichte ist deshalb darauf zu achten, dass es nicht nur eine [...] Geschichte, sondern mehrere Geschichten gibt. [...] Die Deutschen haben deshalb guten Grund, sich mehrerer Identitäten zu vergewissern und auch in ihrem Geschichtsbewusstsein mehrgleisig zu fahren“(A. Assmann 2007: 193 f.).

Die Inklusion der Migrationsgeschichte, darunter der Geschichte der ausländischen Arbeitsmigranten, in das deutsche kulturelle Gedächtnis, die von Assmann im Jahre 2007 noch als Desiderat ausgesprochen wurde, findet gegenwärtig statt. Unter anderem hat mit den 50. Jahrestagen der Anwerbeabkommen der Bundesrepublik mit Italien, Spanien, Griechenland und der Türkei (2005-2011) das öffentliche Interesse an der Geschichte der sog. „Gastarbeiter“ zugenommen. Eine Fülle von Publikationen sowie einige Ausstellungen sind im Zusammenhang mit diesen Jubiläen, vor allem auf der Grundlage lokalhistorischer Recherchen in verschiedenen Orten der Bundesrepublik entstanden.

Auch die Literatur leistet hierzu als wichtiges Medium der Erinnerung ihren Beitrag. Zu den Funktionen von literarischen Texten gehören Astrid Erll zufolge „die Vermittlung von Schemata zur Kodierung von Lebensläufen, die Herausbildung von Vorstellungen über vergangene Lebenswelten, die Zirkulation von Geschichtsbildern, die Aushandlung von Erinnerungskonkurrenzen und die Reflexion über Prozesse und Probleme des kollektiven Gedächtnisses“ (2005: 249). Anders ausgedrückt können literarische Texte als Modelle für das kollektive Gedächtnis (Gedächtnisbildung) und von dem kollektiven Gedächtnis (Gedächtnisreflexion) dienen (nach Erll 2005: 265).

Die Verbindung zwischen dem literarischen Text und der außerliterarischen Erinnerungskultur ist in dem Roman Die Ungehaltenen von Deniz Utlu, einem Autor mit Migrationshintergrund, besonders sichtbar. 
Utlu wurde 1983 in Hannover als Sohn türkischer Einwanderer geboren. Als Zwanzigjähriger gründete er das Kultur- und Gesellschaftsmagazin freitext, das bis 2013 in gedruckter Form erschien. In Berlin und Paris studierte er die Volkswirtschaftslehre. Heute lebt er in Berlin, wo er auch als wissenschaftlicher Mitarbeiter am Deutschen Institut für Menschenrechte tätig ist. Schon während des Studiums und danach war Utlu Kurator von verschiedenen Lesereihen und schrieb Essays, Kurzprosa und Lyrik, die er in Zeitungen, Zeitschriften und Anthologien veröffentlichte. Als Literat erhielt er mehrere Stipendien und Förderpreise. Zusammen mit Marianna Salzmann schrieb er zwei Theaterstücke Tod eines Superhelden (2011) und Fahrräder könnten eine Rolle spielen (2012), die im Berliner Ballhaus Naunynstraße aufgeführt wurden.

Die Ungehaltenen ist Utlus erster Roman. Er erschien 2014 im Graf Verlag und wurde ein Jahr später für das Theater adaptiert. Der Ich-Erzähler und zugleich die Hauptfigur des Romans ist Elyas, ein gebürtiger Berliner aus Kreuzberg, ein erwachsenes Einzelkind von türkischen Einwanderern. Er studiert Jura, bricht jedoch das Studium kurz vor dem Ende ohne klaren Grund ab. Mit Gelegenheitsjobs hält er sich über Wasser. Er besucht regelmäßig seinen Onkel Cemal, hängt mit dem bekannten Kneipenbesitzer Veit und dem türkischen Kumpel aus der Kindheit Hakim rum. Nachdem er auf einer Feier die junge Anästhesistin Aylin kennengelernt hat, fühlt er sich immer mehr durch die Geschichte der türkischen „Gastarbeiter" angesprochen, zu denen sowohl seine als auch Aylins Eltern gehörten. Dann begleitet er die Ärztin auf ihrer Reise in die Türkei, wo sich das Paar näher kommt. Am Ende des Romans kehren beide nach Berlin zurück.

Im Weiteren soll sowohl auf das gedächtnisbildende als auch auf das gedächtnisreflektierende Potenzial des Romans eingegangen werden. Die leitenden Fragen lauten also, was der Text inhaltlich zum gegenwärtigen Bild der Migration im deutschen kollektiven Gedächtnis beitragen kann und welche Stellung er zu dem Wie des Erinnerns, der deutschen Erinnerungskultur, bezieht.

\section{Gedächtnisbildung}

\subsection{Die Geschichte der „Gastarbeiter“}

Im Roman Die Ungehaltenen wird an einen bedeutenden Teil der deutschen Migrationsgeschichte - die Gastarbeiterbewegung - erinnert, die genau zehn Jahre nach dem Zweiten Weltkrieg, mit dem Anwerbeabkommen mit Italien von 1955, begann und die Bevölkerungsstruktur der Bundesrepublik Deutschland bis heute wesentlich prägt. Unter anderem geht die Entstehung der größten ethnischen Minderheit der BRD, der türkischstämmigen Gruppe in Deutschland, hauptsächlich auf diese Einwanderung zurück. Die ersten offiziell eingeladenen Arbeitskräfte 
aus der Türkei kamen 1961 in die BRD (1964 nach West-Berlin), die letzten wurden 1973 angeworben. Bei der Ankunft in Deutschland waren die Arbeitsmigranten der Wirtschaftswunderzeit höchstens vierzig, meistens jünger. Jetzt sind sie schon im Ruhestand, manche leben als Rentner wieder in der Türkei, viele sind bereits gestorben. Somit wäre in jedem Fall die vorher genannte Assmannsche „kritische Schwelle“ von vierzig Jahren überschritten. In diesem Kontext scheint es kein Zufall zu sein, dass die ersten staatlichen Feierlichkeiten in der Bundesrepublik zu einem Jahrestag des deutsch-türkischen Anwerbeabkommens genau vierzig Jahre nach dessen Unterschreiben, im Herbst 2001, stattfanden.

Die erste Generation der türkischen „Gastarbeiter“ in Deutschland, an die bei jenem und späteren Jubiläen erinnert wurde, repräsentieren im Roman die Eltern von Elyas und Aylin. Die Väter haben in den Sechzigerjahren das Angebot der Bundesrepublik in Anspruch genommen und danach jahrzehntelang für deutsche Firmen gearbeitet. Die Darstellung der Beweggründe, die sie zum Verlassen ihrer Heimat brachten, weicht jedoch von dem typischen Bild eines armen Türken ab, dem die Ausreise nach Deutschland die Flucht aus der Lebensmisere und vor der Arbeitslosigkeit ermöglichte sowie die Chance auf einen zivilisatorischen Sprung bot. Elyas Vater musste ins Ausland gehen, da er in der Türkei als regimekritischer Student tätig war, Aylins Vater wurde wegen eines Missverständnisses von der Familie ausgestoßen und dann aus dem Heimatdorf vertrieben. Elyas Mutter arbeitete als Übersetzerin bei der Polizei in Deutschland. Aylins Mutter war dagegen keine „Gastarbeiterin“. Sie hatte ihren zukünftigen Ehemann während seines Urlaubs in Istanbul kennengelernt, war ihm in die BRD gefolgt und führte dort den Haushalt.

In Utlus Roman werden einige Motive thematisiert, die in den Gastarbeiterberichten immer wieder auftreten. Zu diesen Leitmotiven gehört der Rückkehrmythos, die gleichermaßen lange von den Migranten wie der Bundesregierung aufrechterhaltene Vorstellung, dass die ausländischen Arbeitnehmer in einer kurzen Zeit mit dem gesparten Geld in ihre Heimat zurückkehren werden (Utlu 2014: 70, 75, 160). Um den Kontakt zu der Heimat nicht abreißen zu lassen, fuhren die „Gastarbeiter“ regelmäßig in der Urlaubszeit mit Koffern voller Geschenke zu den Verwandten in die Türkei (Utlu 2014: 18). Der Rückkehrplan wurde jedoch, gewöhnlich mit der Geburt der Kinder, von den meisten aufgeschoben und dann ganz aufgegeben. Im Roman erfüllt sich nur Aylins Mutter ihren Rückkehrwunsch und zieht, nachdem ihre Ehe in die Brüche gegangen ist, allein in ihr Herkunftsland um. Die Väter werden erst nach dem Tod in die Türkei zurückgebracht, um dort begraben zu werden.

Neben der gespürten Vorläufigkeit des Aufenthaltes in Deutschland gehörte jahrzehntelang harte Arbeit - körperlich, schmutzig, nicht selten gesundheitsschädlich, in Schichten und im Akkord - zum Alltag der Arbeitsmigranten. Elyas Vater stand bei Ford in Köln am Fließband, um sein Studium der Volkswirtschaftslehre zu finanzieren, Aylins Vater lackierte Drähte. Die Gesundheit dieser Männer wurde durch die Arbeit ruiniert. Im Lebensabend verschlechtert sich ihr Zustand so sehr, dass sie 
ständig auf die Hilfe von Familienangehörigen angewiesen sind. Im ersten Teil des Romans stirbt der krebskranke Vater von Elias, im zweiten der Vater von Aylin.

Im Falle dieser Männer erfüllte sich der Traum von einem besseren Leben im Ausland offensichtlich nicht ganz. Während die Deutschen im Berufsleben einen Aufstieg erlebten und vom Wirtschaftswunder profitierten, mussten sich die „Gastarbeiter" mit recht bescheidenen Wohn- und Lebensbedingungen abfinden. Eine verlassene typische Gastarbeiterwohnung aus den Siebzigerjahren zeigt Elyas seinen deutschen Freunden im Dachgeschoss der Markthalle (Utlu 2014: 15). Yakub Yefa, Aylins Vater, berichtet über seine erste Unterkunft in Berlin, die er mit dreizehn anderen Personen teilte. Als seine Neuvermählte ihm nach West-Berlin folgte, erlitt sie deshalb einen Schock: „Meine Frau hatte sich Deutschland nämlich anders vorgestellt: das dort jeder vierzehn Wohnungen besitzt und nicht umgekehrt jede Wohnung vierzehn Bewohner hat" (Utlu 2014: 159).

Die Ehe scheitert an dem deutschen Alltag. Mit zehn Jahren erlebt Aylin die Trennung ihrer Eltern. Nach dem Beginn von Aylins Studium verlässt ihre Mutter Deutschland, um sich erneut in der Türkei einzurichten. Dass die Auswanderung viele türkische Familien zerstörte, zeigt der Roman auch durch das Prisma der sogenannten „Kofferkinder“. In der Bar seines Freundes trifft Elyas einen alten Mann, der sich auf der Suche nach seinem Enkel befindet. Dieser ist von zu Hause weggelaufen, nachdem er jahrelang zwischen der Türkei und Deutschland pendeln musste (Utlu 2014: 40 ff.). Die vom verzweifelten Großvater erzählte Geschichte steht stellvertretend für das Schicksal von etwa 700.000 türkischen Gastarbeiterkindern, die nach der Auswanderung ihrer Eltern bei Familienangehörigen, bei Omas und Opas, Tanten und Onkeln in der Türkei, in schmerzhafter Trennung oder ständig zwischen zwei Ländern und Kulturen hin- und herfahrend aufwuchsen. ${ }^{2}$ Ein „Kofferkind“ war auch der Regisseur Hakan Savaş Mican, ${ }^{3}$ der zusammen mit Necati Öziri Utlus Roman für das Maxim Gorki Theater adaptiert und dort auf die Bühne gebracht hat (Wildermann 2016).

\subsection{Die Geschichte Kreuzbergs}

Die Ungehaltenen bringt auch ein Stück der Geschichte Berlins, oder genauer gesagt eines Stadtteils Berlins in Erinnerung. Alle Hauptfiguren des Romans wohnen in

${ }^{2}$ Die Geschichte der „Kofferkinder“ wurde über Jahrzehnte sowohl von den betroffenen Personen als auch von deren Familien tabuisiert. In der deutschen Öffentlichkeit ist das Thema seit der Veröffentlichung des Buches von Gülcin Wilhelm Generation Koffer-Die zurückgelassenen Kinder (Berlin 2011) und der Ausstrahlung des Dokumentarfilms von Anke Kültür Kofferkinder. Zurückgelassen in der Türkei (2013) präsent.

3 Von den Kindern der „Gastarbeiter“ der ersten Generation erzählt Hakan Savaş Mican auch in eigener szenischer Installation On My Way Home (2014). 
Kreuzberg, das seit 1961 an drei Seiten von Grenzen zur DDR umgeben war. Elyas, ein gebürtiger Kreuzberger, ist zu jung, um sich an die Mauerzeit zu erinnern. Er gibt zu, ,von der ganzen Ost-West-Geschichte nur David Hasselhof mitbekommen [zu haben], der beim Mauerfall Looking for Freedom gesungen hatte" (Utlu 2014: 14). Die Grenzöffnung und der Fall der Mauer sind dagegen den älteren Kreuzbergbewohnern noch lebhaft in Erinnerung, zum Beispiel die Bilder von den „Ostlern“, die ihr Begrüßungsgeld im „Türken- und Junkyviertel“ abholten (Utlu 2014: 14). Beim Mauerfall wurde auf beiden Seiten der Grenze, auch unter westdeutschen Zuwanderern gejubelt, die negativen Konsequenzen der friedlichen Revolution trafen aber die Letzteren am schwersten. Der Familienfreund, Onkel Cemo, drückt die Enttäuschung seiner Generation über die darauffolgende Entwicklung aus: „Damals war hier vieles anders. Zwei Straßen weiter stand die Mauer. Sie war nicht schön, aber es war ruhiger mit ihr. Wir haben sie niedergerissen. Kaum war sie weg, wollte man, dass auch wir weggingen. Das ganze Land feierte die Einheit, aber niemand fragte uns. Dabei waren wir am meisten betroffen. Wir hatten unsere Jahre an dieser Mauer verbracht" (Utlu 2014: 8). So schlussfolgert er weiter, der Mauerfall habe sie fertiggemacht (Utlu 2014: 22).

Nun werden die Kreuzberger Zeugen einer fortschreitenden Gentrifizierung ihres Stadtteils, der die alten Bewohner mit Wohnpreiserhöhung und Zwangsräumungsurteilen zum Abzug zwingt. Nach fünfunddreißig Jahren müssen der türkische Verein und seine kurdischen Nachbarn ihre Räume verlassen, die ehemalige Fleischerei verwandelt sich in eine Bar, der Teeladen in ein Andenken-Geschäft für Touristen (Utlu 2014: 72, 168 f.). Kreuzberg verwandelt sich von einem Randbezirk zu einer City der Bundeshauptstadt.

An die zunehmende Ausgrenzung auch der alteingesessenen Migranten nach der Wende wird im Roman genauso erinnert wie an die zeitgleiche Stärkung der Berliner Neonazi-Szene. In diesem Zusammenhang werden von Utlu auch die berüchtigten 36 Boys ins Gedächtnis gerufen. Im Roman wird diese gewalttätige Straßengang, die sich nach dem Postzustellbezirk Südost 36, dem SO36, nannte und zu der vor allem türkischstämmige Jugendliche aus Kreuzberg gehörten, als eine Selbstverteidigungsgruppe der Migrantenkinder vorgestellt, die angesichts der Passivität der Polizei ihr Viertel vor Rechtsradikalen schützte (Utlu 2014: 35). Zu der Straßenbande, die seit Ende der Achtzigerjahre bis Mitte der Neunziger aktiv blieb, gehört im Roman der ältere Bruder von Elyas' Kindheitsfreund Hekim.

Hekim tritt nicht in dessen Fußstapfen, knüpft aber dort an, wo die 36 Boys angefangen haben, beim Hip-Hop. Auf die subversive Kraft der jungendlichen Gruppen, die weitgehend ausgeschlossen vom Arbeitsmarkt und von Bildung, mit geringsten Mitteln aus Elementen der afroamerikanischen, deutschen und anatolischen Kultur eine neue Subkultur in Deutschland erfunden haben, verwies Deniz Utlu schon in seinem früheren Essay Ins Herz. Versuch einer Jungs-Geschichte über Hip-Hop und Revolte (Utlu 2010) hin. Im Roman wird an den Begründer der DJ-Crew 36 Boys, den Rapper Attila Murat Aydın alias Maxim 
erinnert (Utlu 2014: 76), der mit dreiunddreißig von einem deutschen Rentner ermordet wurde. $^{4}$

Die Romanfigur Rapper Hakim träumt auch von einer großen Musikkariere, bleibt aber in Kreuzberg stecken. Er wohnt im Herzen des ehemaligen SO36, im langgezogenen Hochhaus mit fast 300 Wohnungen am Kottbusser Tor, dem Kotti. 1974 als Modellprojekt realisiert, verkam das Neue Kreuzberger Zentrum, dann kurz Zentrum Kreuzberg genannt (ZK), schnell zu einem sozialen Brennpunkt und Drogenumschlagplatz, für den es auch heute gehalten wird (Mösken 2016). In Utlus Roman wird nicht nur die unbedachte Sozialpolitik Berlins in den Siebzigerjahren angesprochen - ,sie stopften das Ding voll mit Migranten“ (Utlu 2014: 43) -, sondern auch die frühere Praxis der Hausbesitzer und Stadtverwaltung, Migranten als anspruchslose Zwischenmieter ohne langfristige Wohnperspektive für das „Aufwohnen“ der zum Abriss bestimmten Altbauten zu gewinnen (Düspohl 2012: 147). Onkel Cemo erzählt Elyas, dass die Mitglieder seines türkischen Vereins die alten Häuser mit eigenen Händen wieder aufgebaut haben (Utlu 2014: 21) und er selbst will schon in den Sechzigern ein Teil der Hausbesetzer-Bewegung gewesen sein (Utlu 2014: 31), die die alten Bauten vor dem Abriss im Zuge der damaligen Kahlschlagsanierung gerettet hat und in den Achtzigerjahren für Schlagzeilen in der bundesrepublikanischen Presse sorgte.

Der Roman Die Ungehaltenen nimmt ohne Zweifel Bezug auf den Mythos von Kreuzberg, einem alternativen Lebensraum, charakterisiert durch seine räumliche Abgeschiedenheit und marode Bausubstanz, tut dies aber jedes Mal aus der Perspektive der dort lebenden türkischen Migranten. Über diese Gruppe der Bewohner schrieb Barbara Lang in ihrer Monografie Mythos Kreuzberg. Ethnographie eines Stadtteils (1961-1995): „Sie sind I-Punkt, Vollendung und Abrundung der multikulturell-schrillen Repräsentation des Stadtteils; als eigenes und ernst zu nehmendes Thema bleiben sie in der westdeutschen Medienlandschaft unterrepräsentiert“" (1998: 161). In Langs Untersuchung, die aus Gesprächen mit Kreuzbergern hervorgeht, nehmen jedoch die ausländischen Stadtteilbewohner im Gegensatz zu Utlus Roman - ebenfalls eine Randposition ein.

\section{Gedächtnisreflexion}

\subsection{Migrationsmuseum}

Die Verwandlung Kreuzbergs vom Aussteiger-Mekka zur Yuppie-Town macht im Utlus Roman Onkel Cemo nicht nur über die Zukunft, sondern noch mehr über die Vergangenheit besorgt: „Nach und nach verschwinden alle. Nichts wird bleiben in

${ }^{4}$ Mehr zu der Kreuzberger Hip-Hop-Szene und zu dortigen Straßenbanden siehe: Agabeyoglu 2014, Kap. 5.2. 
dieser Straße. Vierzig Jahre [...] weg“" (Utlu 2014: 21). Er hofft auf die Hilfe der jungen Generation, die Geschichte der türkischen Community in Kreuzberg vor dem Vergessen zu schützen.

Dem Gedächtnisschwund will unter anderem der Rapper Hakim mit eigenen Liedern entgegenwirken, die die Geschichte seiner Stadt, seines Kiezes und seiner Leute erzählen (Utlu 2014: 76 f.). Bei Elyas keimt dagegen das Interesse für das Leben der Generation seiner Eltern erst langsam auf. Cemos Aufforderung, dass die in Deutschland sozialisierten Kinder der Einwanderer jetzt etwas tun, kämpfen, verändern sollen, lehnt er zu Beginn des Romans irritiert ab (Utlu 2014: 32). Erst nach dem Tod seines Vaters verspürt Elyas den Willen, etwas gegen das Vergessen und für die Präsenz der „Gastarbeiter“ im Bewusstsein der Deutschen zu machen. Er kommt auf die Idee, ein Migrationsmuseum zu schaffen.

Neben Bibliotheken und Archiven gehören Museen zu Institutionen des Speichergedächtnisses (A. Assmann 2007: 157). Ihre Aufgabe ist es, Fragmente einer verlorenen Ordnung zu sammeln, zu bewahren, zu erforschen, zu vermitteln und zu präsentieren. In Anlehnung an Krzysztof Pomian deutet Aleida Assmann diese Einrichtung als einen symbolischen Raum, in dem Objekte automatisch zu Zeichenträgern der Vergangenheit werden (2007: 155). Im Museumsraum werden Dinge rekontextualisiert, in einen Zusammenhang gestellt und nicht selten einer übergreifenden Symbolpolitik unterordnet.

Das Museum ist somit kein neutraler Raum der Vermittlung und Popularisierung von Wissen. Bei der Auswahl und der Zusammensetzung der Objekte stellt sich die Frage, was als Teil der Geschichte anerkannt, welche und wessen Geschichte erzählt und im Gedächtnis bewahrt werden soll. Für die Erinnerungswürdigkeit der Objekte sei maßgeblich, inwiefern sie für eine Nation oder eine Epoche als repräsentativ angesehen werden (Kopf 2001: 388; Radoni 2016: 139). Mathilde Jamin (2004: 154) betont, dass die Museumswürdigkeit eines Objekts nicht zuletzt auch eine Frage von Herrschaft und Partizipation ist, da sie wesentlich davon abhängt, ob die soziale Gruppe, deren Überlieferung es entstammt, als geschichtswürdig betrachtet wird.

Ist die Geschichte der Migranten in Deutschland museumswürdig? Ja, sie scheint museumswürdig zu werden. Seit 1991 befürwortet der von vier türkischen Migranten gegründete Verein DOMiD, Dokumentationszentrum und Museum über die Migration in Deutschland e.V., die Errichtung eines zentralen Migrationsmuseums. Es soll der Vermittlung historischer Entwicklungen, dem Abbau von Mythen und Vorurteilen sowie der Etablierung eines neuen multiperspektivischen Geschichtsbildes dienen (DOMiD 2016). Zur Zeit arbeitet DOMiD am Aufbau eines virtuellen Migrationsmuseums. ${ }^{5}$

${ }^{5}$ https://virtuelles-migrationsmuseum.org/. Im April 2015 präsentierte der Verein sein Konzept eines Migrationsmuseums. Es sollte ein eigenständiges Haus an einem zentralen Ort sein und von vielen Akteuren, darunter selbstverständlich Migrantenorganisationen getragen werden. Die Finan- 
Auch der Protagonist von Utlus Roman will die Möglichkeinen des weltweiten Netzes nutzen und ein virtuelles Museum schaffen. In seinem Oral-History-Projekt sollen Erinnerungen von türkischen Einwanderern, deren Kindern und Enkelkindern als Video-Interviews aufgezeichnet und als Clips, sortiert nach Themen, Namen und Orten online gestellt werden. Elyas' Sammlung soll nicht nur der Generation seines Vaters dienen, sondern vielmehr der zweiten, seiner eigenen Generation (Utlu 2014: 85). Die bereits zitierte Mathilde Jamin (2004: 155 ff.) zählt den intergenerationellen Dialog zu den wichtigsten Funktionen eines (Migrations)Museums. Darüber hinaus erwähnt sie folgende Aufgaben einer solchen Institution: Bewahrung der Zeugnisse, Bearbeitung der eigenen historischen Identität der Migranten, symbolische Anerkennung ihrer Zugehörigkeit zur Gesellschaft, Erforschung und Informationsvermittlung an die Herkunftsdeutschen.

In seinem Museumsprojekt will der Protagonist die drei Grundformen der historischen Präsentation, Erzählen, Ausstellen und Inszenieren, kombinieren. Das Inszenieren ist für ihn besonders wichtig. Die Geschichte soll nicht durch eine Anordnung von Objekten im Raum vermittelt, sondern medial dargestellt und gleichzeitig auch an historischen Orten präsentiert werden (mediale und räumliche Inszenierung). Elyas plant eine Launchveranstaltung, auf der die Clips auf Hausfassaden Westberlins projiziert werden (Utlu 2014: 85 f.). Auf diese Weise würde in dem palimpsestartigen Raum der Hauptstadt die Schicht der Gastarbeitergeschichte mit ihren Protagonisten an die Oberfläche kommen. Das Museumsprojekt kommt jedoch im Roman nicht über ein Konzept hinaus, der Grund ist banal: „Ich trank zu viel, kam Tagelang nicht aus dem Bett, und irgendwann fand ich die Idee albern“"(Utlu 2014: 86).

\subsection{Das Jubiläum}

Das Fest wird als primäre Organisationsform des kulturellen Gedächtnissen angesehen (J. Assmann 2007: 57). Es vergegenwärtigt die Vergangenheit und stärkt dadurch die kollektive Identität einer Gruppe, die sich im Bezug auf die Geschichte definiert. Seine ritualisierte und periodische Struktur sichert auch die kollektiven Erinnerungsinhalte. Es kann aber auch als Instrument der Kontrolle und Reglementierung des Gedächtnisses dienen. Eine besondere Bedeutung kommt dem Feiern von Jahrestagen zu. Beate Binder (2001: 290 f.) bezeichnet gefeierte Jahrestage in gegenwärtigen pluralen Gesellschaften als politisch-ästhetische Handlungen, in denen oft öffentliche Debatten über das nationale Selbstverständnis kulminieren. Sie weist auch auf eine mindestens umstrittene Qualität des auf Grund von Jubiläen wiederkehrenden Erinnerns und fragt, ob der Erinnerungsakt als Zeichen eines in der Gesellschaft verankerten Geschichtsbewusstseins oder eher als inhaltsleerer Verweis auf die Vergangenheit zu deuten sei.

zierung des Projekts wurde für das Jahr 2017 vom Bund gesichert, jedenfalls im Rahmen des Deutschen Auswandererhauses in Bremerhaven, was diesen Vorstellungen nicht ganz entspricht. 
Mit ähnlichen Bedenken betritt die Hauptfigur des Romans Die Ungehaltenen den Raum, in dem das fünfzigste Jubiläum des deutsch-türkischen Anwerbeabkommens gefeiert werden soll. Weil Elyas im Internet einen kritischen Text über die Integration veröffentlicht hat, wurde man im Amt des Bürgermeisters auf ihn aufmerksam und schickte ihm eine Einladung zu der Feier. Er hat zuerst nicht vor, der Einladung zu folgen. Im Gespräch mit seiner Mutter schildert er seine Vorbehalte, stößt aber auf wenig Verständnis. Die Anerkennung hat offensichtlich für beide Generationen eine andere Bedeutung. Die Mutter repräsentiert die erste Generation der Einwanderer, die ihre Heimat verließ, um sich und vor allem ihren Kindern neue Perspektiven und ein besseres Leben zu sichern. Diese Gastarbeitergeneration zeichnete Fleiß und geringe Ansprüche aus. Sie empfand auch Deutschland gegenüber eine gewisse Dankbarkeit, eine Chance auf die Realisierung ihrer Pläne zu bekommen. Die Jubiläumsfeier ist aus der Perspektive der Mutter eine Art Würdigung, eine Nobilitierung.

Der Sohn versucht dagegen von Anfang an die Bedeutung der Feier herabzusetzen, was er auch mit seiner lässigen Kleidung zum Ausdruck bringt. Dabei unterstellt er den Organisatoren böse Absichten: „Gleich sollten in einer Fabrikhalle ein paar Hundert türkische Arbeiter und einige B-Promis sich gegenseitig feiern. Wir sind ja so multikulti. Und hinter dieser Fassade verwünschten alle zusammen diese fünfzig Jahre und jedes Jahr, das dazukam“ (Utlu 2014: 97).

Der Mutter zuliebe entscheidet sich der junge Mann trotzdem, an den Feierlichkeiten teilzunehmen. Dort findet er aber seine Vorurteile bestätigt. Noch vor dem Beginn der Veranstaltung beobachtet er eine Szene, die ihn wütend macht. „Ein Bürokrat“ klopft Staub vom veralteten Jackett eines gewissen „Herrn Bulukuluogulu“ und stellt paternalistisch fest: „Da soll mal keiner behaupten, dass es unseren Einwanderern nicht gut gehe bei uns" (Utlu 2014: 100). Der im Auftrag des Senats gedrehte Dokumentarfilm, der auf der Feier gezeigt wird, trägt den Titel Die, die es geschafft haben. Eine Dokumentation über Migranten, die die Chancen Deutschlands zu nutzen wussten. Mit den vier Protagonisten des Films, darunter der Ärztin Aylin, wird auf der Bühne anschließend ein Gespräch geführt. Als Pausenfüller tritt eine türkische Folkloregruppe auf, was Elyas besonders grausam findet und was ihn an die früheren Völkerschauen erinnert. In seinen Augen ist die gesamte Veranstaltung eine nationale „Livesoap, inszeniert für die Ehrenbürger dritter Klasse“ (Utlu 2014: 106) und die fünfzig Jahre, die gefeiert werden, sieht er als eine für seinen Vater und seine Generation verlorene Zeit. Die Willkommensgrüße kamen zu spät. Dem angebotenen Narrativ von Chancen und Gewinn stellt er eine Geschichte von Trennung, entwürdigenden Anwerbeverfahren, schlechten Wohnbedingungen, erschöpfender Arbeit und schmerzvollem Lebensende entgegen. Der junge Mann ist sich sicher, dass ohne die „Gastarbeiter“ die Geschichte der BRD anders gewesen wäre, seine Kosten-Nutzen-Analyse fällt aber zum Ungunsten der Einwanderer aus.

Elyas will Anerkennung, die sich seiner Meinung nach nicht in inhaltsleeren Gesten erschöpft, sondern sich im gegenseitigen Respekt zeigt. Dabei schließt 
sich das Gastarbeiterkind der Forderung an, die die migrantische Hip-Hop Band Microphone Mafia schon 2002 in seinem Song Denkmal im Namen der Vätergeneration formuliert hat:

Gesundheit und Kraft ließen wir am Band zurück,

haben Deutschland mit aufgebaut, sind Teil von diesem von Meisterstück,

sind keine Einwegflaschen, die man nutzt, dann entsorgt.

Meine Jugend ließ ich hier, also bleib ich an diesem Ort.

Wir brauchen keinen Dank, man soll uns respektieren. (zit. nach Saied 2012: 87)

Dass der Mangel an Respekt den gesellschaftlichen Zusammenhalt verhindert oder zumindest schwächt, zeigt der Roman durch die Bezüge sowohl zu vergangenen als auch zu gegenwärtigen Ereignissen. Beispiel dafür sind der Streik der ausländischen Arbeiter bei Ford 1973, der mit dem Sturm der Polizei und Festnahmen der Streikenden endete (Utlu 2014: 67 ff.), der ausländerfeindliche Brandanschlag in Solingen in den frühen Neunzigerjahren, der fünf Menschen ihr Leben kostete (Utlu 2014: 80) sowie die Morde des Nationalsozialistischen Untergrunds (20002007) mit ihren neun Einwanderer-Opfern (Utlu 2014: 137 ff., 153).

\section{Schlussbetrachtungen}

Der Roman Die Ungehaltenen ist eine wichtige und deutliche Stimme der zweiten Generation der Migranten, zu der sowohl der Autor als auch die Hauptfiguren seines Werkes, Elyas, Aylin und Hekim, gehören. In der Frage der Zugehörigkeit beziehen die Protagonisten eine klare Position. Die mehr oder weniger erfolgreichen Kinder der Einwanderer verstehen sich als Teil der heutigen deutschen Gesellschaft, ihre Muttersprache ist Deutsch, sie nennen Berlin ihre Stadt. Ihre Besuche in der Türkei sind keine Heimreisen - wie im Falle ihrer Eltern -, sondern Erkundungsreisen.

Die Zuwendung zu der Lebensgeschichte ihrer Eltern, die eine Migrationsgeschichte ist, hilft der zweiten Generation eine Kontinuität der eigenen Familiengeschichte herzustellen. Von der Position einer generationsbedingten kritischen Distanz gehen die jungen Protagonisten zu der Position der Repräsentanten, ja Anwälte ihrer Eltern über. Sie sprechen im Namen der bisher Sprachlosen, ohne sie -wie dies manchmal die Mehrheitsgesellschaft tut - zu bevormunden. Zuerst hören sie aber den Zeugen zu, die Erzählung über die Migration entfaltet sich aus den in Gesprächen vermittelten individuellen Erinnerungen der Einwanderer.

Das Einbringen der Privatgeschichten in das kollektive Gedächtnis der Deutschen, die Aufwertung und Anerkennung voraussetzt, wird im Roman einerseits mit ihrer sichtbaren Aufnahme in den symbolischen Raum der BRD gleichgesetzt (Migrationsmuseum). Andererseits wird den Formen des ritualisierten öffentlichen Erinnerns (Jubiläum) gegenüber Misstrauen geäußert. 
Die Reflexionen von Elyas und seinen Altersgenossen verbinden sich somit im Text mit erzählten Erlebnissen der ersten Generation der Migranten, die über den Erfahrungs- und Wissensfundus ihrer Kinder deutlich hinausgehen. Ohne die Vielfalt der individuellen Schicksale zu bestreiten, wird im Roman eine Erlebnisund Erinnerungsgemeinschaft inszeniert, die durch eine gemeinsame Vergangenheitsversion, ein gruppenspezifisches Gedächtnis, vereinigt ist. Ihre Geschichte erscheint erzähl- und erinnerungswürdig.

Den Lesern von Utlus Roman wird dabei keine Gegengeschichte dargeboten, sondern eine neue Perspektive auf die jüngste deutsche Geschichte, deren Protagonisten Migranten und Einheimische gleichermaßen sind. Die „Gastarbeiter“ sind demnach ein Teil des deutschen Wirtschaftswunders und des Wiederaufbaus, sollen also auch ein Teil der Erfolgsgeschichte werden und nicht nur eine Fußnote zu ihr oder ein Problemfall.

\section{Literatur}

Agabeyoglu, Nazli Yener (2014): Deutsch-Türkische Rapmusik in Berlin. Soziokulturelle Faktoren bei der Selektion von Rap-Texten und auditiven Produktionsmethoden unter türkischstämmigen Jugendlichen in Deutschland. Berlin.

Assmann, Aleida (2007): Geschichte im Gedächtnis. Von der individuellen Erfahrung zur öffentlichen Inszenierung. München.

Assmann, Jan (2007): Das kulturelle Gedächtnis. Schrift, Erinnerung und politische Identität in frühen Hochkulturen. 6. Aufl. München.

Binder, Beate (2001): Jahrestag. In: Pethes, Nicolas / Jens Ruchatz (Hrsg.): Gedächtnis und Erinnerung. Ein interdisziplinäres Lexikon. Reinbek b. Hamburg. S. 290-291.

DOMiD (Hrsg.): Auf dem Weg, http://www.domid.org/sites/default/files/broschuere_migrationsmuseum.pdf, 28.09.2017.

Düspohl, Martin / Kreuzberg Museum (Hrsg.) (2012): Kleine Kreuzberggeschichte. Berlin.

Erll, Astrid (2005): Literatur als Medium des kollektiven Gedächtnisses. In: Erll, Astrid / Ansgar Nünning (Hrsg.): Gedächtniskonzepte der Literaturwissenschaft: Theoretische Grundlegung und Anwendungsperspektiven. Berlin. S. 249-276.

Jamin, Mathilde (2004): Migrationsgeschichte im Museum. Erinnerungsorte von Arbeitsmigranten kein Ort der Erinnerung? In: Motte, Jan / Rainer Ohliger (Hrsg.): Geschichte und Gedächtnis in der Einwanderungsgesellschaft. Migration zwischen historischer Rekonstruktion und Erinnerungspolitik. Essen. S. 145-157.

Kopf, Christine (2001): Museum. In: Pethes, Nicolas / Jens Ruchatz (Hrsg.): Gedächtnis und Erinnerung. Ein interdisziplinäres Lexikon. Reinbek b. Hamburg. S. 387-389.

Lang, Barbara (1998): Mythos Kreuzberg. Ethnographie eines Stadtteils (1961-1995). Frankfurt am Main/New York.

Mösken, Anne Lena (2014): Zentrum Kreuzberg am Kottbusser Tor. Vom hässlichen Kotti-Koloss zum beliebten Wohnzentrum. In: Berliner Zeitung, 21. November, http://www.berliner-zeitung. de/1546498, Zugang am 26.09.2017.

Radonić, Ljiljana (2016): Der Kampf um das Gedächtnis im Museum. In: Radonić, Ljiljana / Heidemarie Uhl (Hrsg.): Gedächtnis im 21. Jahrhundert. Zur Neuverhandlung eines kulturwissenschaftlichen Leitbegriffs. Bielefeld. S. 137-157. 
Saied, Ayla Güler (2012): Rap in Deutschland: Musik als Interaktionsmedium zwischen Partykultur und urbanen Anerkennungskämpfen. Bielefeld.

Utlu, Deniz (2010): Ins Herz. Versuch einer Jungs-Geschichte über HipHop und Revolte, http:// denizutlu.de/essays/ins-herz/, Zugang am: 25.09.2017.

Utlu, Deniz (2011): Das Archiv der Migration. In: Der Freitag, 31. Oktober, https://www.freitag.de/ autoren/der-freitag/das-archiv-der-migration, Zugang am: 28.09.2017.

Utlu, Deniz (2014): Die Ungehaltenen. München.

Wildermann, Patrick (2015): Wutbürger in Kreuzberg, Der Tagesspiegel, 2. Juni, http://www.tagesspiegel.de/kultur/die-ungehaltenen-im-gorki-studio-wutbuerger-in-kreuzberg/11854856.html, Zugang am: 28.09.2017.

Germanica Wratislaviensia 143, 2018

(C) for this edition by CNS 